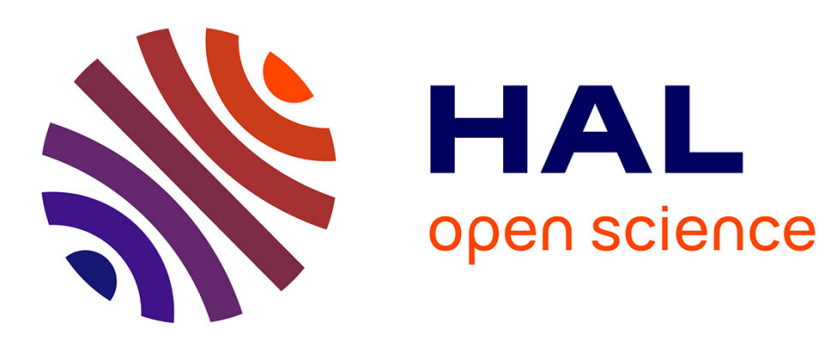

\title{
Virtual cochlear electrode insertion via parallel transport frame
}

Nicolas Duchateau, Nerea Mangado, Mario Ceresa, Pavel Mistrik, Sergio Vera, Miguel Angel Gonzalez Ballester

\section{- To cite this version:}

Nicolas Duchateau, Nerea Mangado, Mario Ceresa, Pavel Mistrik, Sergio Vera, et al.. Virtual cochlear electrode insertion via parallel transport frame. Biomedical Imaging (ISBI), 2015 IEEE 12th International Symposium on, Apr 2015, New York, France. pp.1398 - 1401, 10.1109/ISBI.2015.7164137. hal-01207989

\section{HAL Id: hal-01207989 \\ https://hal.inria.fr/hal-01207989}

Submitted on 1 Oct 2015

HAL is a multi-disciplinary open access archive for the deposit and dissemination of scientific research documents, whether they are published or not. The documents may come from teaching and research institutions in France or abroad, or from public or private research centers.
L'archive ouverte pluridisciplinaire HAL, est destinée au dépôt et à la diffusion de documents scientifiques de niveau recherche, publiés ou non, émanant des établissements d'enseignement et de recherche français ou étrangers, des laboratoires publics ou privés. 


\title{
VIRTUAL COCHLEAR ELECTRODE INSERTION VIA PARALLEL TRANSPORT FRAME
}

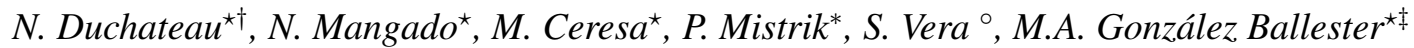 \\ ${ }^{\star}$ Universitat Pompeu Fabra, Barcelona, Spain \\ ${ }^{\dagger}$ Asclepios research Project, INRIA Sophia Antipolis, France \\ ${ }^{*}$ MED-EL, Innsbruck, Austria \\ ${ }^{\circ}$ Alma IT Systems, Barcelona, Spain \\ ${ }^{\ddagger}$ ICREA, Barcelona, Spain
}

\begin{abstract}
We present an automatic, fast and parametrizable algorithm to perform the virtual insertion of a cochlear electrode array into a pre-existent mesh of the human cochlea. Our method reorients the electrode according to the parallel transport frame, a local parameterization of the cochlear centerline directions, robust to the centerline curvature changes. It allows to control the initial roll angle and the extension of insertion from full to partial. Such a virtual insertion, chained with finite element simulations on the electrical activity of the electrode and the cochlear nerves, will enable to test in silico the effects of implant design and positioning on a given patient, and optimize these parameters accordingly.
\end{abstract}

Index Terms - Cochlear implants, virtual deployment, virtual placement, parallel transport frame.

\section{INTRODUCTION}

Sensorineural hearing loss is quickly becoming one of the leading causes of worldwide disabilities. More than 278 million people suffer from moderate to severe hearing disorders due to malformation in the inner ear and $27 \%$ of men and $24 \%$ of women above the age of 45 suffer from a hearing loss of $26 \mathrm{~dB}$ and more [1]. A cochlear implant (CI) is an electronic device which electrode array is surgically placed inside the inner ear, able to directly convert sound into electrical signals. This directly stimulates the auditory nerves and can overcome most of the problems with the transduction in the ear. Despite the success of the technique, the outcomes of CI surgery are very patient-specific and high variability in hearing restoration is observed between patients. One of the possible sources of variation resides in the insertion of the CI electrode array. The result of the stimulation is quite sensitive to the final positioning of the inserted electrodes, especially if residual hearing capabilities are not present [2]. CI electrode insertion has been reported as the most important step in $\mathrm{CI}$

The authors acknowledge the European Union 7th Framework Programme (HEAR-EU FP7-HEALTH-F2-2012-304857).

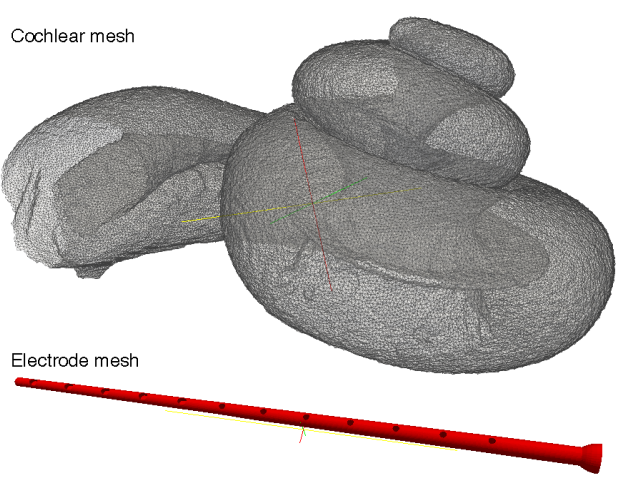

Fig. 1: Example of cochlear mesh extracted from a high-resolution CT, and mesh of the electrode array to be virtually inserted.

surgery, and a high factor conditioning the optimal outcomes $[3,4]$.

The aim of this work is to obtain a fast and automatic patient-specific simulation of the electrode insertion that could be used in finite element simulations (FEM), and therefore help to plan the surgery [5]. To this end, a number of anatomical and insertion parameters make virtual insertion challenging: the anatomical variety of cochlear geometries (in particular, changes in local curvature and number of turns), whether the electrode is fully inserted during the surgery, its initial orientation (roll angle), and the differences between electrode arrays made by different manufacturers.

Patient-specific simulation of CI insertion needs to be easily parameterizable and robust to the above-listed parameters, in order to apply it automatically in a wide range of anatomical and insertion configurations. To the best of our knowledge, this is the first study on automatic simulation of electrode insertion for a FEM study. Up to a certain point, similar concerns and challenges have been reported for the automatic insertion of few other biomedical devices. In particular, in aneurysms treatment, virtual stenting [6] intends to deploy a flow diverter around a vessel centerline, to test the influence of different configurations on a subsequent flow simulation, 


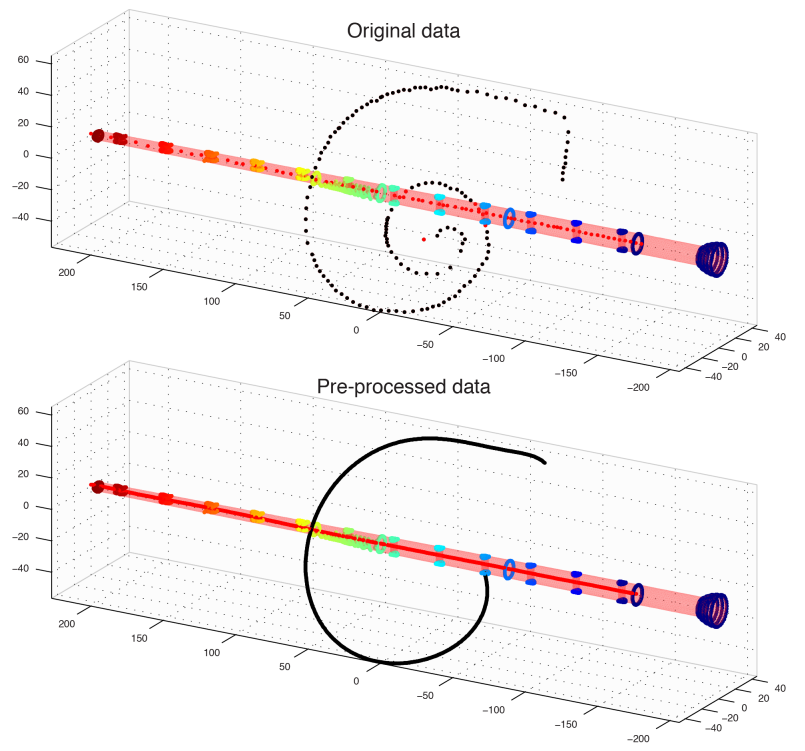

Fig. 2: Cochlear and electrode centerlines (black and red dots, respectively) before and after the pre-processing procedure of Sec.3.1. The points belonging to the electrode mesh are also indicated.

but are based on completely different deployment techniques.

The main contribution of this work resides in the methodology to perform the insertion of a given CI electrode array into any cochlea anatomy. The method is automatic, fast, and easily parameterizable, which are significant advantages for in silico testing a high number of possible configurations to be used in a FEM simulation framework.

\section{METHODS}

In the following, we denote $M_{\text {cochlea }}$ and $M_{\text {electrode }}$ the cochlear and electrode 3D meshes, while $C_{\text {cochlea }}$ and $C_{\text {electrode }}$ refer to their respective centerlines. Examples of these data are given in Figs. 1 and 2.

\subsection{Curve parameterization}

\subsubsection{Tangential, normal and binormal frame $(\mathbf{T}, \mathbf{N}, \mathbf{B})$}

Let's consider a 3D curve parameterized by its length, as $\left\{\mathbf{x}(s) \mid s \in[0,1], \mathbf{x} \in \mathbb{R}^{3}\right\}$. The tangential/normal/binormal frame (also referred to as the Frenet-Serret frame [7, 8]) is defined at each location $s$ of this curve, as:

$$
\begin{aligned}
\mathbf{T}(s) & =\mathbf{x}^{\prime}(s) \\
\mathbf{B}(s) & =\mathbf{T}^{\prime}(s) \times \mathbf{T}(s) \\
\mathbf{N}(s) & =\mathbf{B}(s) \times \mathbf{T}(s),
\end{aligned}
$$

each of these vectors being subsequently normalized.

Notably, these computations involve the curvature vector $\mathbf{T}^{\prime}(s)=\mathbf{x}^{\prime \prime}(s)$, which is not defined on straight portions of a
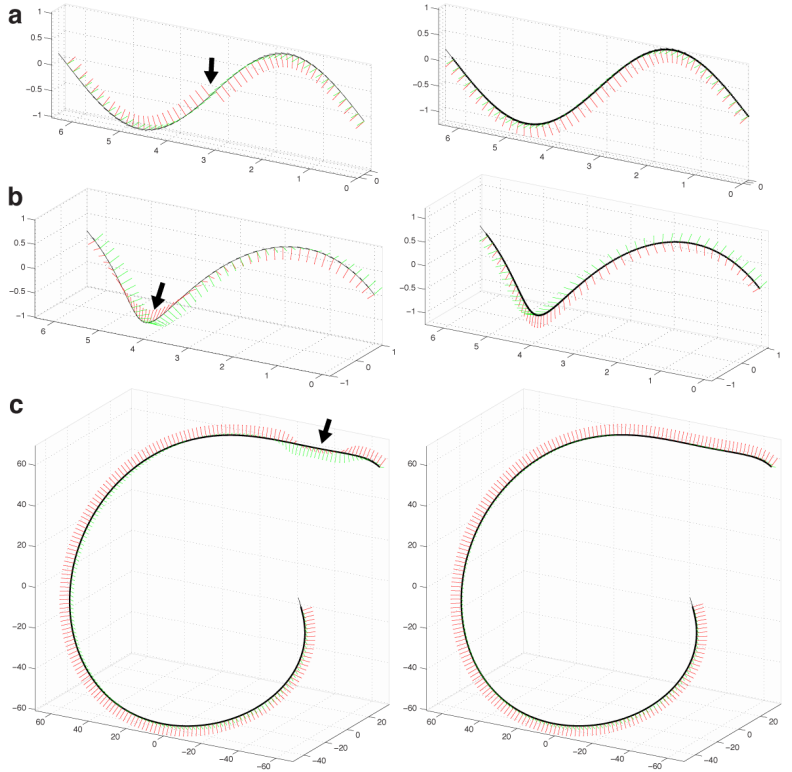

Fig. 3: Illustration of the Frenet-Serret frame [left, $\mathbf{N}$ and $\mathbf{B}$ in red and green, respectively] and the parallel transport frame $\left(\mathbf{T}, \mathbf{E}_{1}, \mathbf{E}_{2}\right)$ [right, $\mathbf{E}_{1}$ and $\mathbf{E}_{2}$ in red and green, respectively] described in Sec.2.1.1 and 2.1.2 on synthetic curves and cochlear centerlines. Arrows point out undesirable changes of local orientation in the Frenet-Serret frame.

the curve, and induces abrupt changes in the orientation of the ( $\mathbf{T}, \mathbf{N}, \mathbf{B})$ frame (left part of Figs.3a, b and c).

\subsubsection{Parallel transport frame $\left(\mathbf{T}, \mathbf{E}_{\mathbf{1}}, \mathbf{E}_{\mathbf{2}}\right)$}

For these reasons, we prefer to rely on the parallel transport frame [7, 8], which results from the parallel transport of a given orthonormal basis $\left(\mathbf{T}(0), \mathbf{E}_{\mathbf{1}}(0), \mathbf{E}_{\mathbf{2}}(0)\right)$ all along the curve. It has the advantage of being robust to the curvature changes that affect the Frenet-Serret frame, and varies smoothly along the curve (right part of Figs.3a, b and c). It is defined as:

$$
\left[\begin{array}{c}
\mathbf{T}^{\prime}(s) \\
\mathbf{E}_{\mathbf{1}}^{\prime}(s) \\
\mathbf{E}_{\mathbf{2}}^{\prime}(s)
\end{array}\right]=\left[\begin{array}{ccc}
0 & k_{1}(s) & k_{2}(s) \\
-k_{1}(s) & 0 & 0 \\
-k_{2}(s) & 0 & 0
\end{array}\right]\left[\begin{array}{c}
\mathbf{T}(s) \\
\mathbf{E}_{\mathbf{1}}(s) \\
\mathbf{E}_{\mathbf{2}}(s)
\end{array}\right],
$$

where $k_{1}$ and $k_{2}$ are the components of the curvature vector with respect to the basis $\left(\mathbf{E}_{\mathbf{1}}, \mathbf{E}_{\mathbf{2}}\right)$.

In practice, this is computed in the following way:

$$
\begin{aligned}
& \mathbf{E}_{\mathbf{1}}(s)=\mathbf{r}(s)-\langle\mathbf{r}(s), \mathbf{T}(s)\rangle \mathbf{T}(s) \\
& \mathbf{E}_{\mathbf{2}}(s)=\mathbf{T}(s) \times \mathbf{E}_{\mathbf{1}}(s),
\end{aligned}
$$

where $\mathbf{r}=\mathbf{R} \cdot \mathbf{E}_{\mathbf{1}}(s-d s), d s$ being an infinitesimal element of length, and $\mathbf{R}$ is the matrix of the $3 \mathrm{D}$ rotation moving $\mathbf{T}(s-d s)$ to $\mathbf{T}(s)$, whose axis is $\mathbf{T}(s-d s) \times \mathbf{T}(s)$, and angle is $\cos \theta=\langle\mathbf{T}(s-d s), \mathbf{T}(s)\rangle$. The computations involving $\mathbf{R}$ are based on the Rodrigues' rotation formula. 

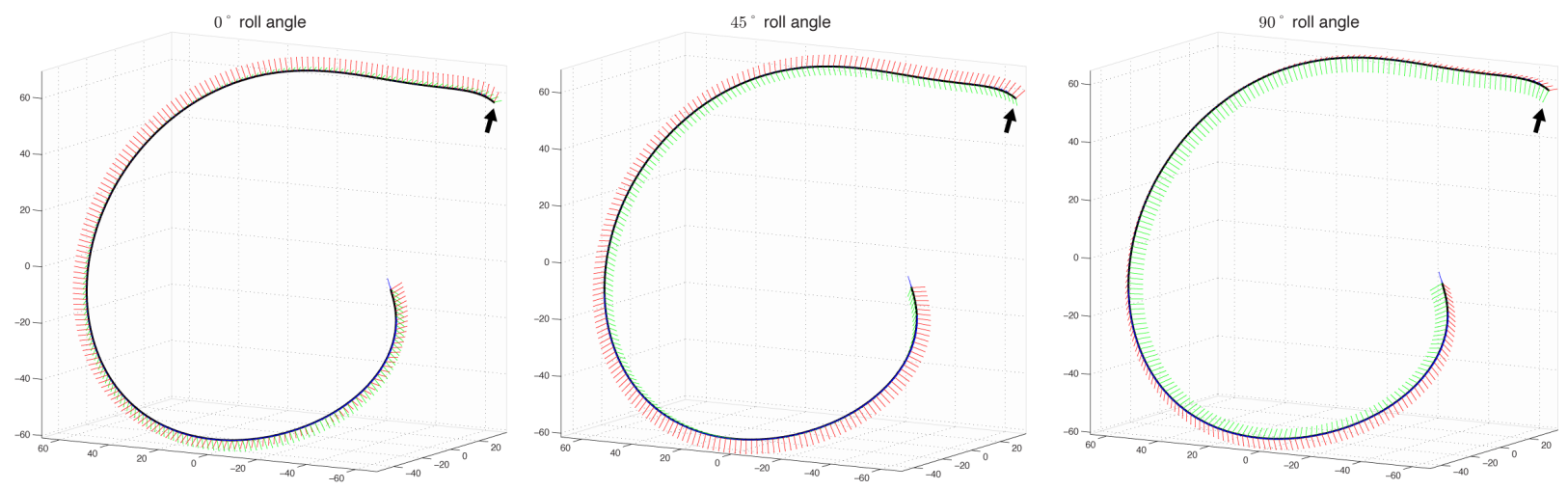

Fig. 4: Different initial roll angles for the parallel transport frame on the same cochlear geometry $\left(0^{\circ}, 45^{\circ}\right.$ and $\left.90^{\circ}\right)$. Red: $\mathbf{E}_{\mathbf{1}}$, green: $\mathbf{E}_{\mathbf{2}}$.

\subsection{Electrode reorientation}

This geometrical process mainly consists in a change of coordinates with respect to the local basis defined at each point of $C_{\text {cochlea }}$. The full process is detailed in Algorithm 1.

The method effectively allows to choose the initial roll angle by which the electrode is introduced (in a pitch-yawroll framework), as illustrated in Fig. $4\left(0^{\circ}, 45^{\circ}\right.$ and $\left.90^{\circ}\right)$. This is simply achieved by properly choosing the initial basis $\left(\mathbf{T}(0), \mathbf{E}_{\mathbf{1}}(0), \mathbf{E}_{\mathbf{2}}(0)\right)$. In our case, $0^{\circ}$ roll corresponds to:

$$
\begin{aligned}
\mathbf{a} & =\mathbf{e}_{x} \times \mathbf{T}(0) \\
\mathbf{E}_{\mathbf{1}}(0) & =\mathbf{T}(0) \times \mathbf{a} \\
\mathbf{E}_{\mathbf{2}}(0) & =\mathbf{T}(0) \times \mathbf{E}_{\mathbf{1}}(0),
\end{aligned}
$$

all of them being subsequently normalized.

\section{RESULTS}

\subsection{Data and pre-processing}

Our methodology is illustrated on three cochlear geometries, obtained from temporal bones excised from human cadavers and scanned with a high-resolution Scanco $\mu$ CT 100 system (Scanco Medical AG, Switzerland). Each image had a nominal isotropic resolution of $24.5 \mu \mathrm{m}$. The data was segmented using semi-automatic techniques as detailed in [9] and [10]. The virtual insertion was tested with a typical electrode array with 12 electrodes (based on MED-EL FLEX Soft design).

In this work, the virtual insertion targets placing the electrode array along the centerline of the cochlea $C_{\text {cochlea }}$. Thus, both $C_{\text {cochlea }}$ and $C_{\text {electrode }}$ need to be determined. This was achieved by parameterizing their corresponding mesh according to a diffusion process, and extracting the centers of mass of each subset of vertices associated to a given diffusion time. Note that this is not the exact location where a real electrode would be in contact with the cochlear anatomy, nor the exact anatomical location of $C_{\text {cochlea }}$, located through the round window into scala tympani along the lateral wall. The simpler centerline used in the current paper was retained for the proof of concept of the virtual insertion, which is still valid for more realistic anatomical locations, targeted in further work.

Both $C_{\text {cochlea }}$ and $C_{\text {electrode }}$ were then resampled to 100 points, uniformly distributed along their length. This was achieved using kernel ridge regression with an exact matching formulation [11], and served to prevent from bias in the subsequent computations about length correspondence.

The amount of points along $C_{\text {cochlea }}$ that serve for support to the insertion of $N \%$ of the electrode array was then determined using length correspondence (proportionality law), and $C_{\text {cochlea }}$ was cut accordingly.

Finally, a second resampling was applied to increase the number of points along each centerline (in our implementation, up to 1000 points uniformly distributed along its length), using the same algorithm as for the first regression. This new resampling served to minimize approximation errors when looking for the points of the centerline that are the closest from a given point of the electrode mesh (Sec.2.2 and Step 1 in Algorithm 1).

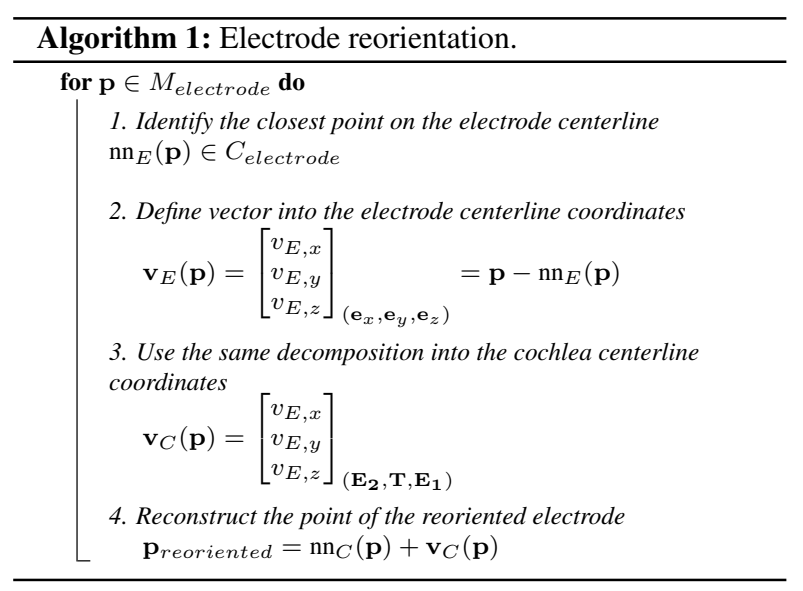



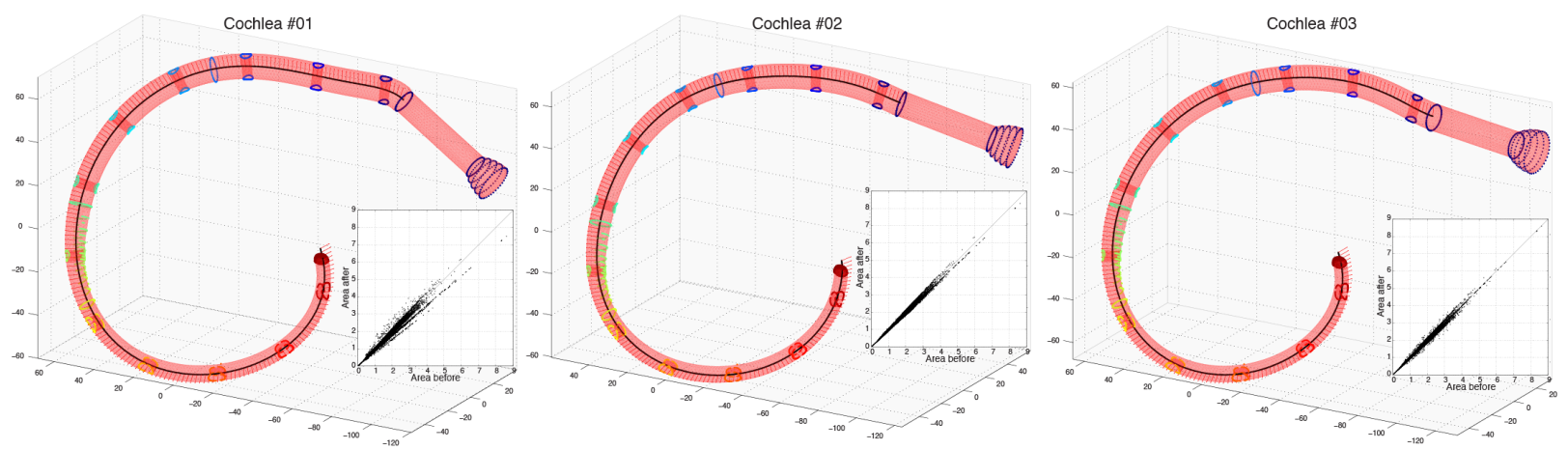

Fig. 5: Full electrode insertion in 3 different cochlear geometries. Red arrows indicate the direction of $\mathbf{E}_{\mathbf{1}}$. The inserted graphics plot the area of each triangle of $M_{\text {electrode }}$ before vs. after the insertion.

\subsection{Examples}

The method takes less than $1 s$ on a non-dedicated standard personal computer. Figure 5 illustrates the insertion results achieved for the three different cochleas mentioned in Sec.3.1. Full insertion of the same electrode was tested in each case. Complementarily, Fig. 6 illustrates the partial insertion results achieved for the first cochlea, up to $70 \%$ insertion. Note that, as theoretically expected, this methodology introduces little or no distortion in the different subparts of the electrode mesh (different subgroups of color in the electrode points of Figs.2, 5 and 6), as illustrated in the inserted plots in Figs.5 and 6. The method is purely geometric, which contrasts with the potential use of non-linear registration for a similar purpose (e.g. surface and landmark matching as in [8]), in addition to their computational time and possible inaccuracies. However, local mesh folding could occur in cases where the radius of curvature of $C_{\text {cochlea }}$ is smaller than the radius of $M_{\text {cochlea }}$. While this is not the case in the dataset we tested, further work may consider regularization or diffeomorphic constraints to prevent from these artifacts. More physically-related virtual deployment techniques may also be considered, if the gain in accuracy they represent is not overwhelmed by the complexity of their implementation.

\section{CONCLUSIONS}

In this work, we presented a fast, parameterizable insertion algorithm that enables the use of realistic cochlear implant geometries in our previous finite element model of cochlear electrical stimulation. This contribution has potential to provide clinicians and implant manufacturers a better understanding of the impact of implant design and insertion, and to optimize them in a patient-specific framework.

\section{REFERENCES}

[1] World Health Organization, "Deafness and hearing impairment," 2012.

[2] World Health Organization, "The global burden of disease: 2004 update," 2008.

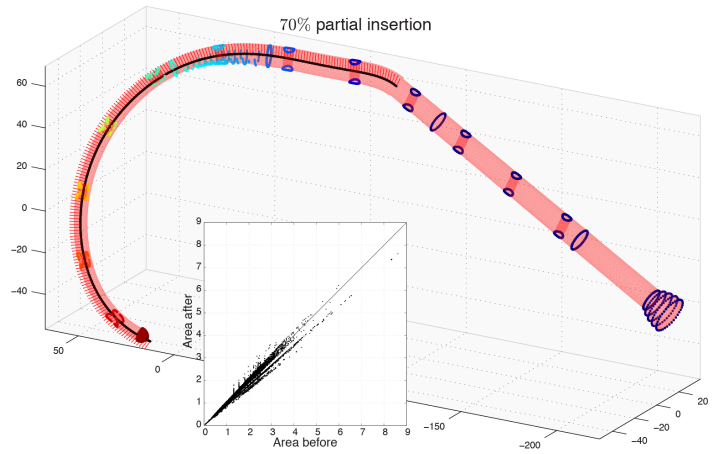

Fig. 6: Partial insertion $(70 \%)$ on the first cochlear geometry. Red arrows indicate the direction of $\mathbf{E}_{\mathbf{1}}$. The inserted graphic plots the area of each triangle of $M_{\text {electrode }}$ before vs. after the insertion.

[3] JT Jr Roland, "Cochlear implant electrode insertion," Oper Tech Otolaryngol Head Neck Surg, vol. 16, pp. 86 - 92, 2005.

[4] M Gani, G Valentini, A Sigrist, et al., "Implications of deep electrode insertion on cochlear implant fitting," J Assoc Res Otolaryngol, vol. 8, pp. 69-83, 2007.

[5] M Ceresa, N Mangado, H Dejea, et al., "Patient-specific simulation of implant placement and function for cochlear implantation surgery planning," in Proc. MICCAI, LNCS, 2014, vol. 8674, pp. 49-56.

[6] I Larrabide, M Kim, L Augsburger, et al., "Fast virtual deployment of self-expandable stents: method and in vitro evaluation for intracranial aneurysmal stenting," Med Image Anal, vol. 16, pp. 721-30, 2012.

[7] RL Bishop, "There is more than one way to frame a curve," Am Math Monthly, vol. 82, pp. 246-51, 1975.

[8] H Bogunovic, JM Pozo, R Cardenes, et al., "Automated landmarking and geometric characterization of the carotid siphon," Med Image Anal, vol. 16, pp. 889-903, 2012.

[9] M Ceresa, W Nogueira, N Carranza, et al., "Patient-specific electrical simulation of cochlear implants for surgical planning and optimization of stimulation patterns," in Proc. CARS. Springer, 2014.

[10] HM Kjer, M Ceresa, N Carranza, et al., "Cochlear finite element modelling: Mesh quality under ssm-driven deformations," in Proc. MeshMed-MICCAI. Springer, 2013.

[11] N Duchateau, M De Craene, G Piella, et al., "Constrained manifold learning for the characterization of pathological deviations from normality," Med Image Anal, vol. 16, pp. 1532-49, 2012. 